\title{
Understanding COVID-19 vaccine hesitancy
}

\author{
A new study unpacks the complexities of COVID-19 vaccine hesitancy and acceptance across low-, middle- and \\ high-income countries.
}

\section{Shingai Machingaidze and Charles Shey Wiysonge}

\section{A} s of 29 June 2021, there had been more than 181 million reported infections with SARS-CoV-2 and nearly 4 million reported deaths from COVID-19 ${ }^{1}$. In May 2020, the 73rd World Health Assembly issued a resolution recognizing the role of extensive immunization as a global public-health goal for preventing, containing and stopping transmission of SARS-CoV-2 ${ }^{2}$. Globally, there are now more than 125 vaccine candidates, 365 vaccine trials ongoing, and 18 vaccines against COVID-19 approved by at least one country ${ }^{3}$. Published research carried out largely in high-income countries cites concerns about the safety of vaccines against COVID-19, including the rapid pace of vaccine development, as one of the primary reasons for hesitancy ${ }^{4}$, but data from low- and middle-income countries (LMICs) have been limited. In this issue of Nature Medicine, Solis Arce et al. present data that begin to address this research gap ${ }^{5}$.

The reluctance of people to receive safe and recommended available vaccines, known as 'vaccine hesitancy', was already a growing concern before the COVID-19 pandemic ${ }^{6}$. A framework developed from research done in high-income countries, called 'the 5C model of the drivers of vaccine hesitancy', provides five main individual person-level determinants for vaccine hesitancy: confidence, complacency, convenience (or constraints), risk calculation, and collective responsibility ${ }^{7,8}$. Promoting the uptake of vaccines (particularly those against COVID-19) will require understanding whether people are willing to be vaccinated, the reasons why they are willing or unwilling to do so, and the most trusted sources of information in their decision-making. Solis Arce et al. investigated these questions using a common set of survey items deployed between June 2020 and January 2021, across 15 studies carried out in Africa, South Asia, Latin America, Russia and the United States ${ }^{5}$.

Included in the analysis were seven studies in low-income countries (Burkina Faso, Mozambique, Rwanda, Sierra

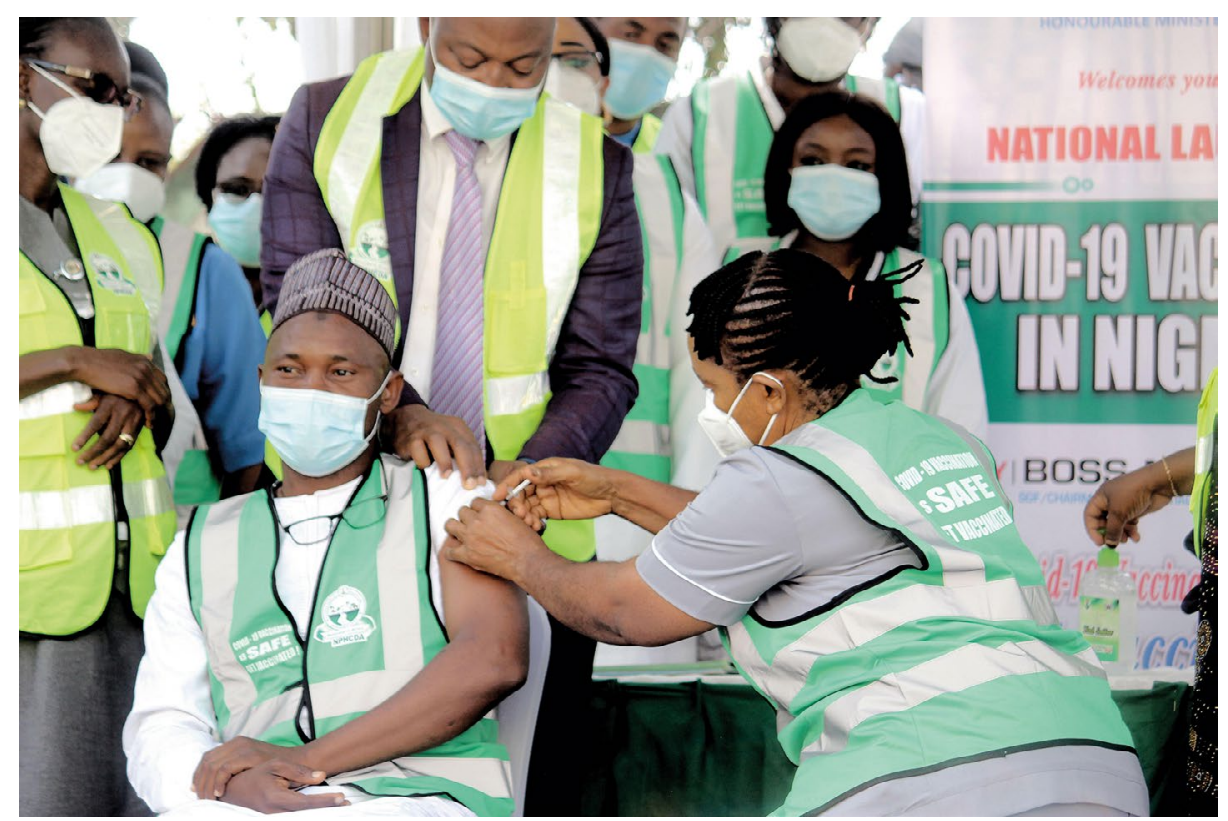

Credit: Majority World CIC / Alamy Live News

Leone and Uganda), five studies in lower-middle-income countries (India, Nepal, Nigeria and Pakistan) and one study in an upper-middle-income country (Colombia). The authors compare these findings with those from two countries at the forefront of vaccine research and development: Russia and the United States ${ }^{5}$. Overall, they found that the average acceptance rate across the full set of studies in LMICs was $80.3 \%$, with lowest acceptance in Burkina Faso (66.5\%) and Pakistan (66.5\%); moreover, the acceptance rate in every sample from LMICs was higher than that of samples from the United States $(64.6 \%)$ and Russia $(30.4 \%)^{5}$. The data show that vaccine acceptance is explained mainly by an interest in personal protection against COVID-19, whereas concerns about side effects are the most common reasons for hesitancy, and health workers are the most trusted sources of guidance about vaccines against COVID- $19^{5}$. It is, however, important to note that reported intentions may not always translate into vaccine uptake?
Another survey was conducted by the Africa Centres for Disease Control and Prevention, in partnership with the London School of Hygiene and Tropical Medicine, between August and December 2020, in 15 African countries (Burkina Faso, Côte d'Ivoire, Democratic Republic of the Congo, Ethiopia, Gabon, Kenya, Malawi, Morocco, Niger, Nigeria, Senegal, South Africa, Sudan, Tunisia and Uganda $)^{10}$. Again, it was found that the majority of respondents in Africa (79\%) would be vaccinated against COVID-19 if it were deemed safe and effective $^{10}$. Perhaps it may be that lived experience in LMICs, where many vaccine-preventable infectious diseases are still causing thousands of deaths annually, results in higher perceived need for or value of vaccines. In contrast, high-income countries have successfully eliminated or eradicated numerous vaccine-preventable diseases and, as a consequence, many people, including medical professionals, have not seen the devastating effects of these diseases in their respective 
countries. This could lead to complacency, altered risk calculations and limited collective responsibility about vaccination decision-making.

With the wide availability of smartphones, more people can now access the internet and social media in LMICs. Although this can be a great tool for self-education, which is a key component of vaccination decision-making, it also presents several challenges in the form of misinformation (including 'anti-vaxx' messaging) and incomplete information, as well as inconsistent and complicated scientific information that may be difficult to understand.

The reasons for COVID-19 vaccine acceptance and hesitancy remain complex. As new SARS-CoV-2 variants emerge, adding further complexity ${ }^{11}$, and new vaccines come to the market, it will be important to maintain a delicate balance in communicating what is known and acknowledging the uncertainties that remain. Researchers and pharmaceutical manufacturers should be as forthcoming as possible, with research data on vaccines against COVID-19 made readily available. International medical journals should ensure that the use of 'expedited reviews' does not compromise the robustness of the peer-review process of key publications on the safety and efficacy of vaccines, or related research findings. Governments should be transparent about their COVID-19 response programs and vaccine availability and should disclose how key decisions are being made. Reporting of adverse events after immunization is a key component of monitoring the implementation of vaccination programs, and although it is important for these events to be documented and reported, intensive media coverage may also discourage people from being vaccinated. The media should therefore report in a responsible and transparent manner, providing clear and unbiased information to its audiences. Finally, people using the internet and social media (including scientists and clinicians) should do so responsibly to avoid spreading falsehoods or using language that could be misinterpreted and could thereby potentially add to vaccine hesitancy.

Although issues of vaccine-distribution equity remain a considerable challenge for LMICs that require urgent intervention ${ }^{12}$, the lag in the rollout of vaccines against COVID-19 in these regions does present a window of opportunity for addressing issues of hesitancy. The findings from Solis Arce et al. ${ }^{5}$ suggest that prioritizing distribution of vaccines to LMICs is justified not only on equity grounds but also on the expectation of higher marginal returns in maximizing global coverage at

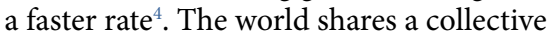
responsibility in fighting this pandemic; therefore, continued research on COVID-19 vaccine acceptance and hesitancy should be a priority. Such research should then be used to inform contextualized campaigns and information-sharing that will ultimately result in increased confidence in and uptake of available vaccines.
Shingai Machingaidze $\mathrm{e}^{1,2,4 凶}$ and Charles Shey Wiysonge 2,3,4 $^{\text {Coun }}$

${ }^{1}$ European and Developing Countries Clinical Trials Partnership, Africa Office, Cape Town, South Africa. ${ }^{2}$ School of Public Health and Family Medicine, Faculty of Health Sciences, University of Cape Town, Cape Town, South Africa. ${ }^{3}$ Cochrane South Africa, South African Medical Research Council, Cape Town, South Africa. ${ }^{4}$ These authors contributed equally: Shingai Machingaidze, Charles Shey Wiysonge.

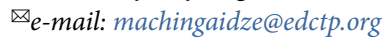

Published online: 16 July 2021

https://doi.org/10.1038/s41591-021-01459-7

References

1. World Health Organization. https://covid19.who.int/ (accessed 30 June 2021).

2. World Health Organization. https://apps.who.int/gb/ebwha/ pdf_files/WHA73/A73_R1-en.pdf (19 May 2020).

3. McGill COVID19 Vaccine Tracker Team. https://covid19. trackvaccines.org/vaccines/ (accessed 27 June 2021).

4. Wouters, O. J. et al. Lancet 397, 1023-1034 (2021).

5. Solis Arce, J. S. et al. Nat. Med. https://doi.org/10.1038/s41591021-01454-y (2021).

6. MacDonald, N. E. Vaccine 33, 4161-4164 (2015).

7. Betsch, C. et al. PLoS One 13, e0208601 (2018).

8. Wiysonge, C. S. et al. Hum. Vaccin. Immunother. 8, 1-3 (2021)

9. Mceachan, R., Conner, M., Taylor, N. \& Lawton, R. Health Psychol. Rev. 5, 97-144 (2011).

10. Africa Centres for Disease Control and Prevention. https://africacdc.org/news-item/majority-of-africans-wouldtake-a-safe-and-effective-covid-19-vaccine/ (17 December 2020).

11. Abdool Karim, S. S. \& de Oliveria, T. N. Engl. J. Med. 384, 1866-1868 (2021)

12. United Nations. https://news.un.org/en/story/2021/04/1089972 (16 April 2021).

\title{
Tumor-infiltrating lymphocytes make inroads in non-small-cell lung cancer
}

\author{
A phase 1 trial shows promising anti-tumor activity of tumor-infiltrating-lymphocyte therapy in patients with \\ advanced non-small-cell lung cancer.
}

Joshua R. Veatch, Sylvain Simon and Stanley R. Riddell

\footnotetext{
pithelial cancers are driven by somatic mutations and aberrantly expressed genes. These genetic alterations can encode peptide sequences that are presented on the tumor cell surface by major histocompatibility complex molecules, and these complexes can be recognized by the
}

patient's T cells. Tumor-reactive T cells that infiltrate the tumor often become dysfunctional and fail to eradicate the tumor. Immune-checkpoint blockade (ICB) can improve $\mathrm{T}$ cell function and bring about tumor regression ${ }^{1}$, but unfortunately, most patients either fail to respond or develop resistance over time. An alternative strategy for boosting anti-tumor immunity is to isolate functional tumor-infiltrating lymphocytes (TILs) from resected tumors or tumor biopsies and expand them ex vivo for adoptive transfer back into the patient. This approach has led to durable remissions 